\title{
Mitigating Motion Sickness in Automated Vehicles with Vibration Cue System
}

This paper was downloaded from TechRxiv (https://www.techrxiv.org).

LICENSE

CC BY-NC-SA 4.0

SUBMISSION DATE / POSTED DATE

08-01-2022 / 11-01-2022

CITATION

Li, Daofei; Chen, Linhui (2022): Mitigating Motion Sickness in Automated Vehicles with Vibration Cue System. TechRxiv. Preprint. https://doi.org/10.36227/techrxiv.18092492.v1

$\mathrm{DOI}$

10.36227/techrxiv.18092492.v1 


\title{
Mitigating Motion Sickness in Automated Vehicles with Vibration Cue System
}

\author{
Daofei $\mathrm{Li}^{\text {a, }}{ }^{\text {, Linhui Chen }}{ }^{\text {a }}$ \\ ${ }^{a}$ Zhejiang University, Institute of Power Machinery and Vehicular Engineering, Faculty of Engineering,
}

Hangzhou, 310027, China.

\begin{abstract}
Motion sickness is very common in road transport. To guarantee ride comfort and user experience, there is an urgent need for effective solutions to motion sickness mitigation in semi- and fully-automated vehicles. Considering both effectiveness and user-friendliness, a vibration cue system is proposed to inform passengers of the upcoming vehicle movement through tactile stimulation. By integrating the motion planning results from automated driving algorithms, the vibration cueing timing and patterns are optimized with the theory of motion anticipation. Using a cushion-based prototype of vibration cue system, 20 participants were invited to evaluate this solution in two conditions of driving simulator experiments. Results show that with the proposed vibration cue system, it could also help participants to comprehend the cues and to generate motion anticipation. The participants' motion sickness degrees were significantly lowered. This research may serve as one foundation for the detailed system development in practical applications.
\end{abstract}

\section{Key words}

automated vehicle, motion sickness, driving simulator, vibration cue, motion anticipation

\section{Practitioner Summary}

In automated vehicles, passengers engaging in non-driving tasks are apt to severe motion sickness. A vibration cue system and cueing strategy are proposed and optimized to inform passengers of the upcoming vehicle movement. Simulator experiments of 20 participants proved its effectiveness in promoting motion anticipation and reducing motion sickness.

\section{Introduction}

Car sickness is one of the most common forms of motion sickness, which happens to a large group of passengers. Sickness symptoms, including nausea, pallor, cold sweating and vomiting, lead to unpleasant rides, e.g., comfort and customer experience. Motion sickness is a difficult problem both for theoretical and engineering researches, which needs contributions from multi-disciplines, e.g., psychology, human factors and ergonomics, mechanical and control engineering, etc.

As for motion sickness theory, there have been many researches on how and why motion sickness happens. On one hand, Reason proposed the neural mismatch hypothesis that motion sickness is due to sensory rearrangement, i.e. motion sickness happens when there is a difference between the current visual-vestibular-proprioceptive stimulus

1 Corresponding author. Email address: dfli@zju.edu.cn (Daofei Li)

This article has been accepted for publication in Ergonomics, published by Taylor \& Francis. 
inputs and those expected on the basis of previous sensory inputs ('exposure history') (Reason, 1969). Reason further specified protective adaptation and a neural mismatch model of motion sickness. It can explain in more details the process of motion adaptation/sickness between sensor input and internal neural store (Reason, 1978). To describe the connection between sensory conflict and motion sickness, Oman combined the observer theory from control engineering and proposed a more specific mathematical model of sensory conflict hypothesis (Oman, 1990). Based on the sensory rearrangement theory, Bos et al. proposed the subjective vertical conflict model, which believes that motion sickness originates from the difference between the 'subjective vertical' determined by the current and the expected sensory inputs (Bles et al., 1998; Bos and Bles, 1998). On the other hand, Riccio and Stoffregen presented a postural instability theory, which believes that prolonged postural instability is the cause of motion sickness symptoms (Riccio and Stoffregen, 1991). Dong et al. further applied the postural instability theory in explaining the 'driver-passenger effect', i.e. drivers are responsible to control the vehicle movement, so they can effectively stabilize their bodies by using anticipatory control, while passengers can only achieve less accurate posture stability via compensatory control and thus are more likely to suffer from motion sickness (Dong et al., 2011).

With the rapid development of automated driving technology, it is foreseeable that highly automated vehicle is not far away for the general public. Driving automation can relieve drivers from driving tasks and may even completely transform them into passengers. As visioned in futuristic designs, the cockpit arrangement of self-driving cars, including seats, windows and screens, will be revolutionized. Passengers will be able to engage in various non-driving tasks (NDTs), e.g. reading, surfing the Internet, watching movies, video meeting, etc. However, such engagement of NDTs may aggravate their motion sickness, since less attention is paid to the driving and road conditions (Pfleging et al., 2016; Sivak and Schoettle, 2015). For satisfactory user experience, motion sickness will face a bigger challenge in future automated vehicles (Diels and Bos, 2016; Wada, 2016).

To address the challenge, the motion sickness problem can be tackled through the physical environment aspect (i.e. via minimizing the vehicle motion stimuli) or/and the human passenger aspect (i.e. via helping passengers accommodate the motion stimuli). On one hand, to actively mitigate motion sickness in automated vehicles, the uncomfortable motion stimuli can be minimized or even removed by improving motion planning and control algorithms. For example, algorithms of speed planning (Wada, 2016), path planning and steering control (Ukita et al., 2020) were proposed to minimize motion sickness. In our previous studies, a motion planning algorithm is developed via frequency shaping approach ( $\mathrm{Li}$ and $\mathrm{Hu}, 2021)$. In another research, we included the motion sickness model into the automated car-following algorithm design (Li et al., 2021). However, in some driving scenarios, e.g., automatic emergency braking, these active mitigation approaches should be compromised for safety and traffic efficiency considerations. On the other hand, if providing human passengers with the useful information about the environment or vehicle movement, it is hopeful to help them generate motion anticipation and thus better accommodate the motion stimuli. This can be derived from the above two theories of motion sickness mechanism. According to sensor conflict theory, such information of future vehicle motion can help the passenger's internal neural store to generate anticipation that matches the upcoming stimulus (Reason, 1978). Based on the postural instability theory, such reminding information will make passengers more likely to apply anticipation control and thus achieve better posture stability like drivers (Dong et al., 2011; Chang et al., 2021).

To this end, there have been some studies on optimizing ergonomic design to mitigate passenger motion sickness. 
Miksch et al. presented a system using the live video of the road ahead as a background for reading (Miksch et al., 2016). Van Veen designed a proximal peripheral light signal system, which can enhance passengers' awareness of the traffic situation (van Veen et al., 2017). Karjanto et al. designed a peripheral visual feedforward system to improve the situation awareness of passengers for non-driving tasks (Karjanto et al., 2018). Kuiper et al. proposed an auditory cue method to suggest forward or backward motion to improve passengers' anticipation to motion (Kuiper et al., 2020a,b). However, these informing approaches, either visual or auditory, may interfere with the non-driving tasks of passengers (e.g. reading or video watching), which will limit their applications in future automated vehicles. On the other hand, comparing to visual or auditory cueing, the vibrotactile cueing approach is promising as a better solution, since it can elicit faster reactions of passengers while bringing less interferences with non-driving tasks (Meng and Spence, 2015).

In this paper, we propose a vibration cue array system to be mounted in the seat-cushion of automated vehicles. It aims to remind the occupants of the upcoming movement and thus to mitigate motion sickness. With a fabricated prototype, we carry out simulator experiments, and verify whether the system can effectively promote motion anticipation and reduce motion sickness without much disturbance to the ride or non-driving tasks.

\section{Materials and methods}

\subsection{Vibration cue System}

\section{(1) Cueing-based mitigation in automated vehicles}

Figure 1 schemes the cueing-based mitigation approach for motion sickness in automated vehicles. Usually, the basic algorithm modules of automated driving include sensing, predicting, planning and control. Based on sensed and predicted information of traffic environment, the planning module will calculate an optimized path and a speed profile in the coming steps, to achieve both safety and comfort. Such planned path and speed profile will be accurately tracked in the control module, by applying appropriate traction, braking and steering inputs. Given the planned motion trajectory in future steps, the cueing system can promote the generation of motion anticipation. Therefore, the cueing provided to human passengers should include the key information of future vehicle motion stimulus, including its direction and magnitude within a given time horizon.

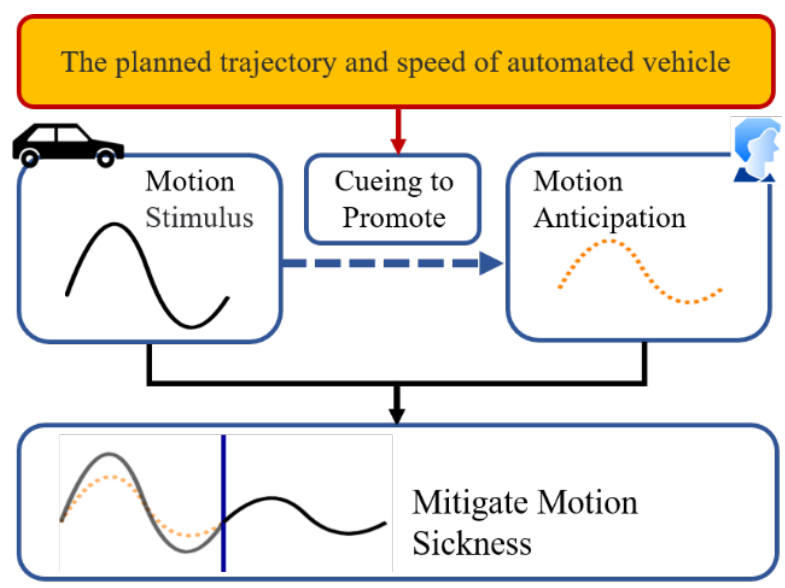

Figure 1 Motion anticipation to mitigate motion sickness 


\section{(2) Vibration cueing and prototype design}

Different from audio or visual cues used in previous studies, here a vibration cue system is proposed, which applies tactile stimulation to promote the passenger (participant) to generate motion anticipation in automated vehicles. Without visual or audio attention needs, the vibration cue is expected to have less disturbance to passenger activities.

A prototype of seat cushion with vibration cueing is designed and fabricated, as shown in Figure 2. On the inner layer of the cushion, 16 groups of vibrating motors, i.e., totally 32 motors, are arranged into a 4 by 4 array. Each motor group can be independently controlled, to provide different types of cueing, including timing and vibration patterns. For example, the direction of linear and rotational motion can be mapped to a specific sequence of motor on/off activation. The magnitude of motion can be mapped to the vibration intensity of motors, which is similar to that of a mobile phone. In this preliminary study, we focus on the lateral motion stimulus and its corresponding cueing strategy.

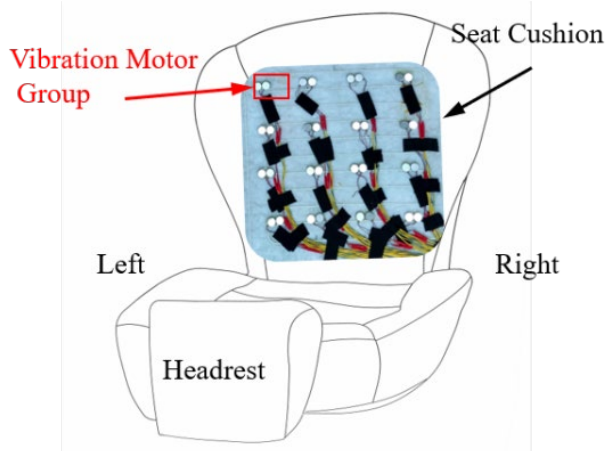

Figure 2 Prototype of vibration cushion

\section{(3) Cueing strategy}

Figure 3a schemes how the vibration cue system works in an example driving scene. The ego car initially drives at a constant speed in the left lane. A rapid lane change trajectory to the right lane is planned to avoid colliding with the oncoming traffic cones. According the motion planning algorithm of automated driving, we can directly know the lane-change initial point and the future motion stimulus level in this planned manoeuvre. The vibration cue system determines and applies the corresponding cueing pattern, including the cueing initial point and its cueing form.

By defining TTC (the Time To lane-Change initial point), the vibration cueing initial point is determined as the vehicle position at TTC $=1.2 \mathrm{~s}$, i.e., the cueing starts 1.2 seconds before the vehicle motion. The cueing form for an upcoming motion stimulus corresponds to four different vehicle lane-changing operations. For example, the rapid left lane change operation corresponds to the fast burst of motor vibration cue, like a wave from right to left, as shown in Figure 3b. The slow right lane change operation corresponds to a slow burst vibration pattern shifting from left to right.

In detail, for the fast vibration cue from left to right, each row of motor groups vibrates from left to right successively starting from TTC $=1.2 \mathrm{~s}$. Each row vibrates for $0.2 \mathrm{~s}$, and a cue lasts for $0.8 \mathrm{~s}$ in total, as shown in Figure $3 \mathrm{~b}$. For the slow vibration cue from right to left, each row of motor vibrates from right to left successively from TTC $=1.2 \mathrm{~s}$. Each row vibrates for $0.3 \mathrm{~s}$, and a cue lasts for $1.2 \mathrm{~s}$ in total. The software of the vibration cue system is implemented in MATLAB, including cueing location calculation, cueing form decision, and control sequence generation. 


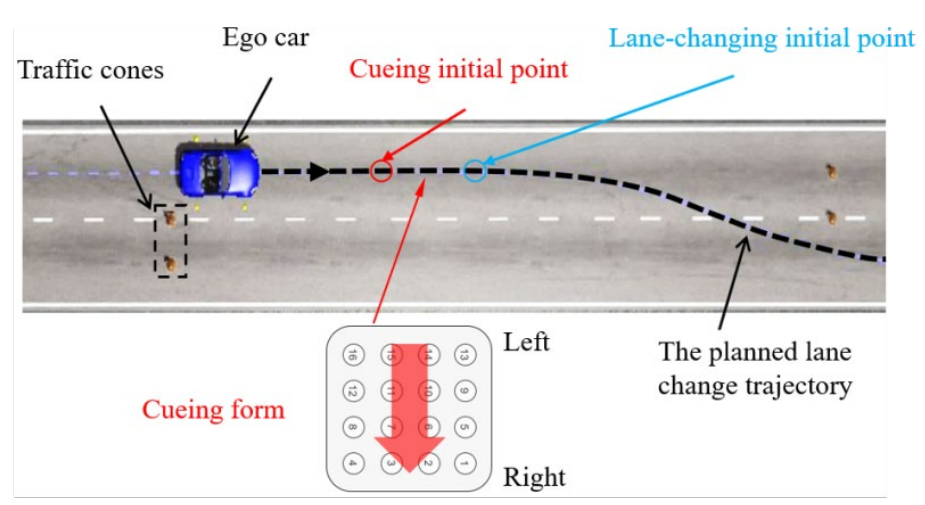

(a) Vibration cue system and experiment scene

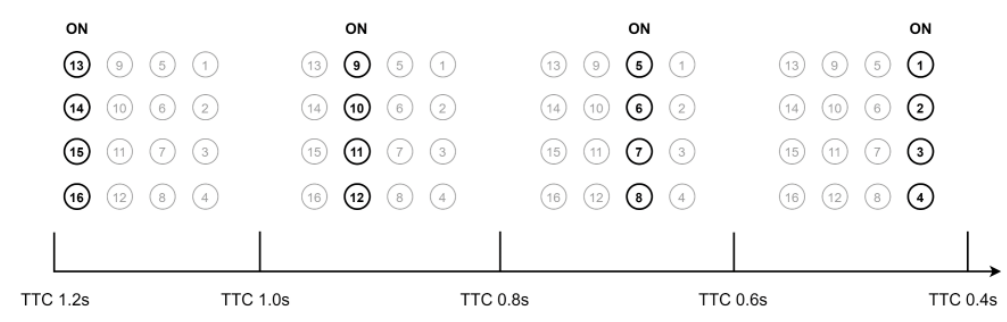

(b) Fast vibration from left to right

Figure 3 The principle scheme of vibration cue system

\subsection{Participants}

The study invited 20 healthy subjects ( 19 males and 1 female), with age ranging from 21 to 39 (mean $=23.10$, SD =4.02). 17 of them hold driver licenses, and all of them had more than 5 years of vehicle riding experience. Before the experiment, the participants' motion sickness susceptibility was analysed via the MSSQ-short questionnaire. The average MSSQ-Short score was $12.06 \pm 6.14$, which was close to the average score 12.9 \pm 9.9 in Golding's study (Golding, 2006).

None of the participants had motion sickness related diseases, and their visual acuity or corrected visual acuity was normal. Participants were asked not to drink or take drugs for 24 hours before the experiment. In addition, participants were advised to get enough sleep the day before the experiment to ensure that they were physically and mentally normal at the beginning of each experiment.

\subsection{Experimental Setup}

The vehicle driving context was set as a series of consecutive lane-changing manoeuvres at medium speed. It can mimic aggressive driving styles in urban traffic. Note that such consecutive lane-changing setting may not represent common daily driving, it can be viewed as the accelerated testing of our vibration cue system, especially testing whether passengers can respond correctly and timely to the cueing information. The detailed setup is given as follows.

(1) Road. Based on the vehicle dynamics simulation software MSC Carsim, a straight one-way two-lane road was built. Figure 4 presents one example scene, in which the vehicle encounters obstacles (traffic cones) and plans to change to the left lane. The planned vehicle trajectory in the $5,000 \mathrm{~m}$ long road, as shown in Figure 5a, was tracked using a path-follow controller. The centre line equations of left and right lanes were $\mathrm{Y}=1.75 \mathrm{~m}$ and $-1.75 \mathrm{~m}$, respectively. Totally 75 pairs of traffic cones were setup, and a total of 76 lane change operations were required. In 
addition, every five pairs of traffic cones were joined as a group, and there was a certain interval between each group.

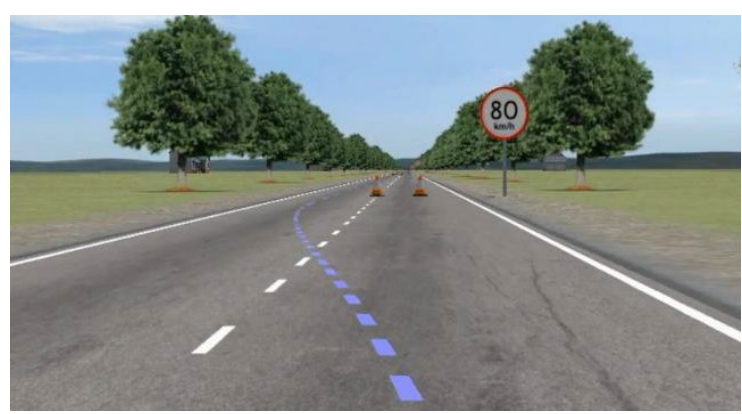

Figure 4 An example driving scene

(2) Speed. The speed of ego car was planned as follow: 1) to accelerate from stationary to $40 \mathrm{~km} / \mathrm{h}$ in $30 \mathrm{~s}$, 2) to complete 76 lane change operations at a constant speed, and 3) to slow down from $40 \mathrm{~km} / \mathrm{h}$ to $0 \mathrm{~km} / \mathrm{h}$ in $30 \mathrm{~s}$. The speed was tracked with a closed loop controller. The entire $5,000 \mathrm{~m}$ trip took about 7 minutes to finish.

(3) Path. Two kinds of lane changing operations were set, i.e., the rapid and slow ones, as shown in Figure 5b. The order of rapid and slow lane change manoeuvres was random, and thus their corresponding motion stimuli were unpredictable for participants. Additionally, the setting of different lane change trajectory and lane change timing were used to verify whether the different forms of vibration cue were distinguishable by participants.

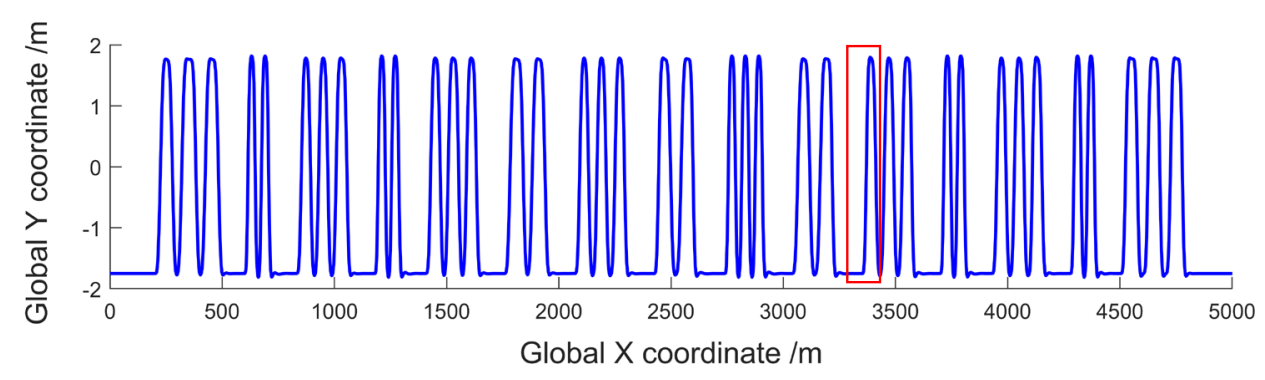

(a) vehicle trajectories

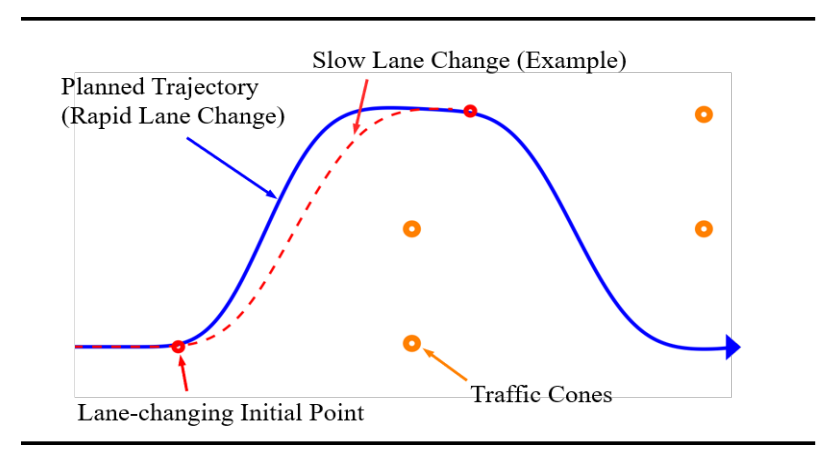

(b) rapid and slow lane-changes

Figure 5 Planned vehicle trajectories in experiment

\subsection{Apparatus}

A six-degree-of-freedom (6-DOF) driving simulator platform was used to simulate real ride conditions, as shown in

Figure 6. The platform was equipped with operable steering wheel, gas and brake pedal, monitor, audio, etc. The simulator was surrounded by black curtains, forming a closed environment to control the experiments without any 
interference. Simulation experiment scene in Carsim and vibration cue system in MATLAB/Simulink were running in the host computer. The ECG data was collected using the multi-channel physiological system BioPac MP160 and its acquisition software module. The host computer and real-time computer NI-PXI-8135 performed the calculation of the simulation scenes and the vibration cue system, and outputted the corresponding motion to the 6-DOF platform. The calculated vibration cues were applied. Pedal opening, steering angle and ECG data were recorded in the host computer.

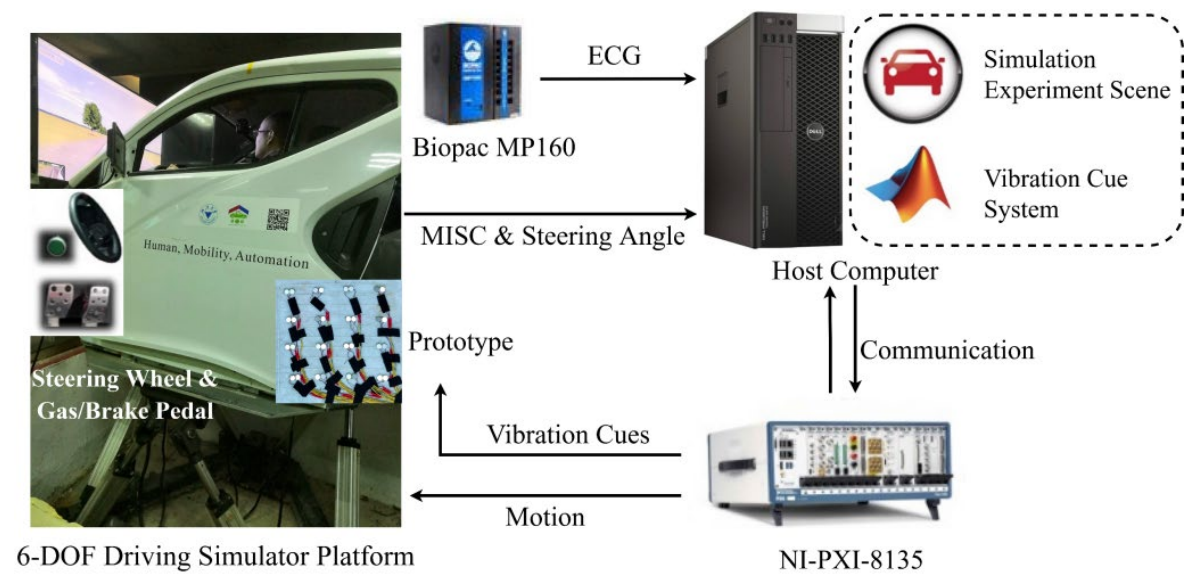

Figure 6 Simulator and algorithm computers

\subsection{Procedure}

The experiment of this study was divided into two conditions. In the experimental condition, vibration cue system was activated on to promote their motion anticipation. In the control condition, vibration cue system did not work, and the participants were expected to have no motion anticipation. These two conditions adopted the same simulation experiment scene and applied the same motion stimuli of lane changes. The vehicle completed two same stages of $5,000 \mathrm{~m}$ driving, taking $14 \mathrm{~min}$ in total. There was a non-stimulation period of about one minute between these two driving stages, which was used to reset the experimental scene. During the experiment, all monitors were turned off and the ambient light was adjusted to avoid external visual stimulus.

(1) Time. All participants completed both the experimental and control conditions. The interval between the two conditions was at least 24 hours, to give participants sufficient time to eliminate the motion sickness caused in the first experiment condition. To avoid the influence of motion anticipation (due to their familiarity with the scene) on the results, all subjects participated this research for the first time and did not understand the design details of the experiment. Additionally, for each participant, the order of the two conditions is randomly set rather than fixed.

(2) Preparation phase. During the preparation phase, participants read the instructions to understand the purpose and procedure of the experiment, and signed an informed consent form. The participants were instructed that the steering wheel was not used for driving or changing the direction of movement, but only as one way of expressing their answers, like a group of buttons to push in common human factors experiments. After that, participants were equipped with physiological data acquisition equipment. Additionally, in the experimental condition with cueing, participants were given 7 minutes to familiarize themselves with the vibration cue system.

(3) Formal experiment. In the formal phase of the experiment, participants, as passengers of ego car, did not participate in vehicle handling. Participants first sat in the driver's seat for at least 3 minutes to adjust their physical 
and mental states. Then the ego car started and automatically drove and changed lanes, to give participants motion stimulation through the 6-DOF driving simulator platform. During the experiment, participants were asked to sit in a natural pose with seatbelt buckled up, and to look at the shut-off screen in front, which was to further limit the participants' head movement and visual stimulation. Participants in both conditions were required to evaluate the degree of their own motion sickness. If the subjects had significant discomfort symptoms during the procedures, the experiment was immediately terminated. In the experimental condition with cueing on, participants were required to operate the steering wheel according to the perceived vibration cues to report their motion anticipation.

After the experiment, the participants stayed in the simulator for $2 \mathrm{~min}$ and then filled in the questionnaire.

\section{Results}

\subsection{Motion sickness degree}

Participants evaluated their own motion sickness degree based on the 11-point Misery Scale (MISC) (Bos et al., 2005). The symptoms of each degree correspond to the score on a scale of $0-10$. The larger the score, the higher the degree of motion sickness. The scoring was carried out using two pedals in the simulator. Each experiment was scored 30 times (i.e., 30 grading points), each taking an interval of about 30 seconds.

The values of MISC in the whole process of the experiment were analysed by two-way ANOVA, and the results showed that there was a significant difference in the degree of motion sickness between the two conditions $(\mathrm{F}=12.755$, $\mathrm{P}=0.002$ ). Mean values and standard errors of MISC in two conditions are shown in Figure 7. The scores of the control condition grew faster, and the scores of most points in the control condition were higher than those in the experimental condition. In other words, in the control condition (without vibration cue system), the motion sickness degree of participants accumulated at a faster rate.

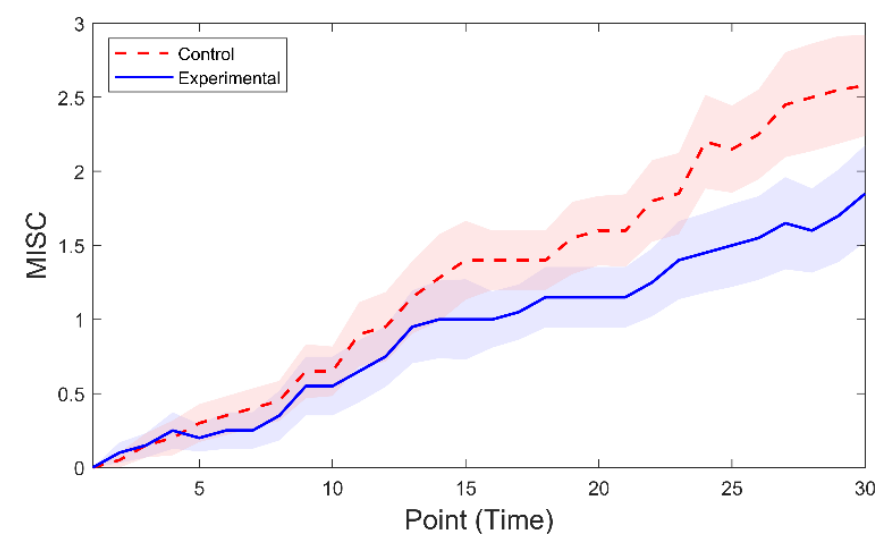

Figure 7 Mean value ( \pm standard error) of MISC

Figure 8 shows the numbers of participants with MISC values reaching 3 and 4 in both conditions (i.e. mild and moderate discomfort symptoms), respectively. The red solid/dotted lines are for the control condition, and the blue solid/dotted lines are for the experimental condition. Note that there was a slight decrease in MISC between grading points 15 and 17, which was due to the non-stimulation period between the two experiment segments. For most points, the number of participants who reached scores 3 and 4 in the control was more than that in the experimental. In the final grading point, the number in control was also larger. The above results are consistent with the results of ANOVA and mean values of MISC, all illustrating the differences between the two conditions. The overall results show that 
the vibration cue system can effectively mitigate the motion sickness of participants.

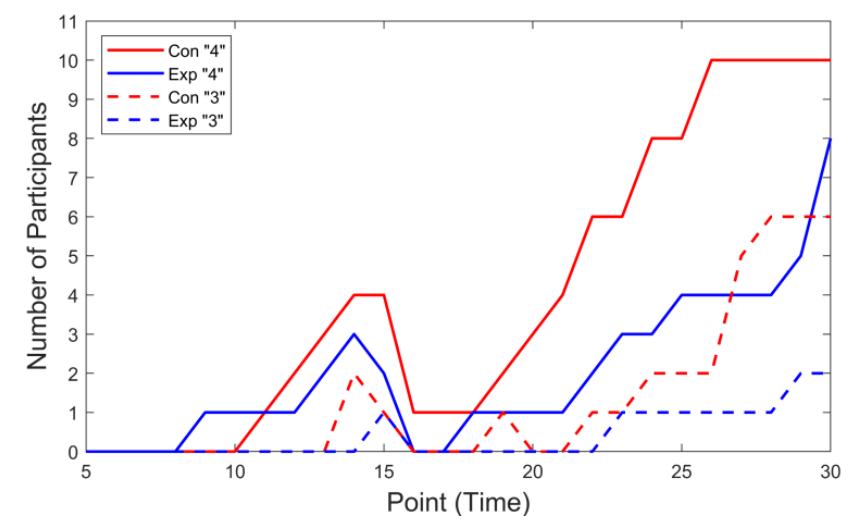

Figure 8 Participant numbers with MISC reaching 3 and 4

\subsection{Motion anticipation}

Participants operated the steering wheel to represent their motion anticipation. After perceiving and comprehending the vibration cues and identifying the vehicle's lane change operation, they needed to turn the steering wheel immediately. Rapid lane change to the left corresponds to a left turn steering wheel with a large angle (suggested value of $\pm 180^{\circ}$ ). Slow lane change to the right corresponds to a right turn steering wheel with a small angle (suggested value of $\pm 90^{\circ}$ ).

Figure 9 shows the steering angle of a participant, which is obviously divided into 15 lane-change groups and 4 kinds of angle. Among them, the positive peak (e.g. $60 \mathrm{deg}, 150 \mathrm{deg}$ ) corresponds to turning left. For this participant, the motion anticipation represented by steering angle was consistent with the actual vehicle movement. Notice that among the total 6 lane-changes in Group 13, the $5^{\text {th }}$ lane-change to left used the rapid trajectory option, while the other five times used slow change, i.e., the participant accurately identified the lane-changes by perceiving different cueing forms.

The steering angle of all participants was analysed. For most participants, steering angle was consistent with the actual vehicle movement. Statistics show that the average success rate for all participants reaches $89.59 \%$, while the lowest is $68.42 \%$. This shows that the participants could clearly distinguish the direction and intensity of cueing, which could further promote appropriate motion anticipation.

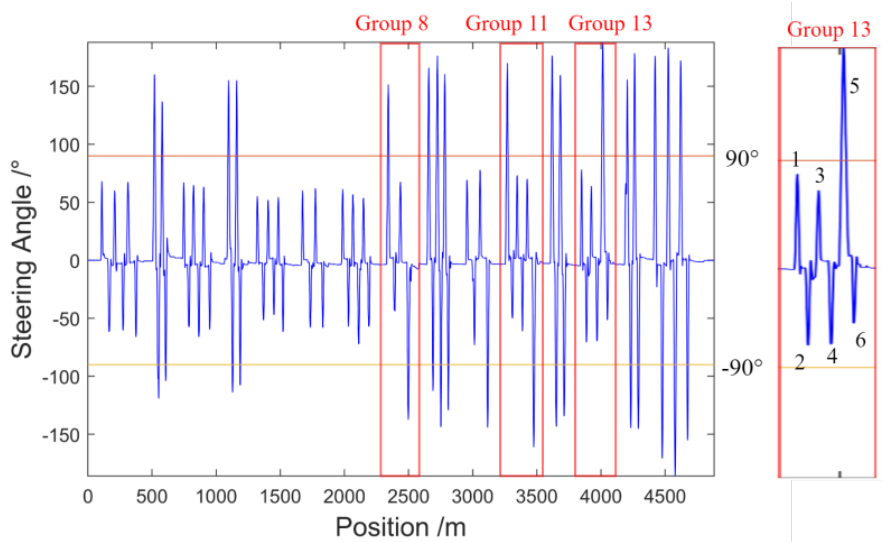

Figure 9 Steering angle of a participant during a 5,000m trip 


\section{$3.3 \mathrm{HRV}$}

Heart rate variability (HRV) method can be used to analyse the influence on the autonomic heart rate regulation mechanism of participants (Malik et al., 1996; Malińska et al., 2015). A previous study showed that the low frequency (LF $0.04-0.15 \mathrm{~Hz}$ ) component and the LF/HF ratio of HRV grow as the increase of the motion sickness degree, while the high frequency (HF 0.15-0.40Hz) component decreases (Lin et al., 2011).

The HRV data of two 7 min rides (stage 1 and stage 2) in each condition were analysed. The LF/HF ratio in the two conditions is shown in Figure 10, i.e., $\operatorname{Exp} 1(\mathrm{M}=0.76, \mathrm{SD}=0.21)$, Exp $2(\mathrm{M}=0.97, \mathrm{SD}=0.36)$, Con $1(\mathrm{M}=1.28$, $\mathrm{SD}=0.92)$, and Con $2(\mathrm{M}=1.07, \mathrm{SD}=0.47)$. Nonparametric tests were performed and reported in Table 1 . The results show that the LF / HF ratio of Con 1 and Con 2 was slightly higher than that of Exp 1 and Exp 2, and the ratio of Exp 1 was the lowest. However, no significant difference between the two conditions was found.

Table 1 Nonparametric tests of LF/HF ratio

\begin{tabular}{ll}
\hline Test & p value \\
\hline Exp 1 - Exp 2 & 0.134 \\
Con 1 - Con 2 & 0.935 \\
Exp 1 - Con 1 & 0.126 \\
Exp 2 - Con 2 & 0.719 \\
\hline
\end{tabular}

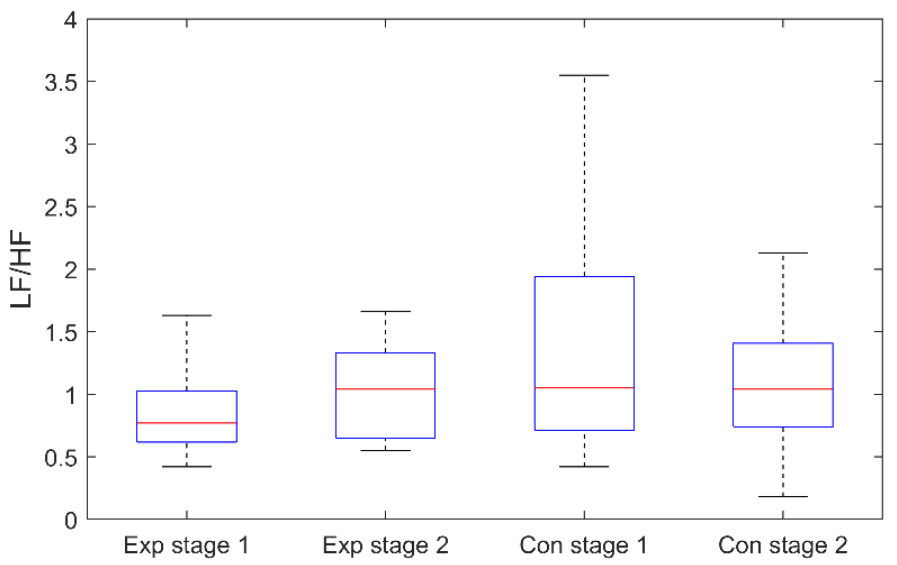

Figure 10 The LF/HF ratio under two experiment conditions

\subsection{Questionnaire}

The questions and results for both conditions are summarized in Table 2. Since in the control condition the vibration cue system was off, only questions 4-5 were filled to assess participants' anticipations for the motion. The second column of Table 2 presents answers to the questions and the corresponding score, and the third and fourth columns contain the mean values and standard deviations of the scores in the two conditions.

Questions 1-2 were designed to evaluate the effectiveness of vibration cues. Results show that the participants give high scores and thought that the vibration cue was easy to perceive, understand and identify. Question 3 was related to the vibration cue timing design, and the most participants thought the time was reasonable. But some participants 
believed that the vibration cue time (location) was too late. Questions 6 and 7 were about the user experience on the vibration cue system. Participants believed that the vibration cue had a slight impact on the ride experience, but considering its functionality, it was feasible for the vehicle to equip with the vibration cue system. Questions 4-5 were about passengers' motion anticipation, for which the Mann-Whitney U test was performed on the results of the control and experimental conditions. Results indicate that there was a significant difference in question 4 $(\mathrm{p}=0.006<0.05)$, but no significant difference in question $5(\mathrm{p}=0.146)$.

Table 2 Questionnaire after the experiment

\begin{tabular}{|c|c|c|c|}
\hline Question & Score & Control & Experimental \\
\hline $\begin{array}{l}\text { 1. Perceptibility of the vibration } \\
\text { cues? }\end{array}$ & $\begin{array}{l}\text { 0: imperceptible } \\
\text { 5: perceptible }\end{array}$ & I & $\begin{array}{l}M=4.70 \\
S D=0.66\end{array}$ \\
\hline $\begin{array}{l}\text { 2. Comprehensibility of the } \\
\text { vibration cues? }\end{array}$ & $\begin{array}{l}\text { 0: incomprehensible } \\
\text { 5: comprehensible }\end{array}$ & I & $\begin{array}{l}\mathrm{M}=4.45 \\
\mathrm{SD}=0.60\end{array}$ \\
\hline $\begin{array}{l}\text { 3. The vibration cues timing is } \\
\text { reasonable? }\end{array}$ & $\begin{array}{l}\text { 0: early; } 3 \text { : reasonable } \\
6 \text { : late }\end{array}$ & I & $\begin{array}{l}\mathrm{M}=3.35 \\
\mathrm{SD}=1.09\end{array}$ \\
\hline $\begin{array}{l}\text { 4. Predictability of the upcoming } \\
\text { motion? }\end{array}$ & $\begin{array}{l}\text { 0: unpredictable } \\
\text { 5: predictable }\end{array}$ & $\begin{array}{l}\mathrm{M}=2.25 \\
\mathrm{SD}=1.44\end{array}$ & $\begin{array}{l}\mathrm{M}=3.60 \\
\mathrm{SD}=0.88\end{array}$ \\
\hline $\begin{array}{l}\text { 5. Perceptibility of the lane } \\
\text { changing speed? }\end{array}$ & $\begin{array}{l}\text { 0: imperceptible } \\
\text { 5: perceptible }\end{array}$ & $\begin{array}{l}M=3.44 \\
S D=1.26\end{array}$ & $\begin{array}{l}\mathrm{M}=4.10 \\
\mathrm{SD}=0.97\end{array}$ \\
\hline $\begin{array}{l}\text { 6. Does the vibration affect the } \\
\text { ride? }\end{array}$ & $\begin{array}{l}\text { 0: negative impact } \\
\text { 5: no impact }\end{array}$ & 1 & $\begin{array}{l}\mathrm{M}=3.50 \\
\mathrm{SD}=1.05\end{array}$ \\
\hline $\begin{array}{l}\text { 7. The necessity of the vibration cue } \\
\text { system? }\end{array}$ & $\begin{array}{l}0: \text { unnecessary } \\
\text { 5: necessary }\end{array}$ & l & $\begin{array}{l}\mathrm{M}=3.30 \\
\mathrm{SD}=1.13\end{array}$ \\
\hline
\end{tabular}

\section{Discussion}

\subsection{Experiment design}

In this study, the experimental scenes and the movement of automated vehicles were set up based on lane change manoeuvre, which implies the main motion stimulus is lateral acceleration. This is similar to previous researches, in which either lateral or longitudinal stimuli are setup (Karjanto et al., 2018; Kuiper et al., 2020). In real application, there are usually stimuli from multiple directions even in flat road driving. Although our simulator supports coupled lateral and longitudinal movements, this one-axis stimulus setup can keep the participants' head movement in a limited range and thus improve the controllability of experiments. In spite of this, it is still interesting to further explore the cueing effects in more complex movement situations.

Most participants hold driver licenses, and the rest of them also have sufficient vehicle ride experience, so it can be considered that they were familiar with the lane changing behaviours. Here we used driver steering wheel as the interface for reporting passengers' motion anticipation, rather than by giving oral/handwritten responses, or hitting a button. Such interface is intuitive and straightforward in the experiment context, which can encourage the participants to perceive and understand cues and to output the motion anticipation on this basis. The success rate of motion anticipation generation implies that the experiment controllability was guaranteed, i.e., although participants could 
not perfectly perceive and understand the given cues, they did pay attention to the cues by reporting.

\subsection{Cueing strategy}

Interestingly, after the experiment some participants reported that 'by steering operations, although not involving in the real vehicle motion control, they felt like they were manipulating the vehicle through the steering wheel and receiving the corresponding motion stimulus'. This sense of 'manipulation' is similar to the 'sense of agency' (David et al., 2008). On one hand, in theory, this active 'manipulation' facilitates the participant's role transition from a pure passive passenger to a driver that actively engages in driving tasks, which plays a certain role in reducing motion sickness (Rolnick and Lubow, 1991). According to the postural instability theory and also the 'driver-passenger effect', by the role transition to a 'driver', the participant can perform anticipatory control for better postural stability, thus for less motion sickness. On the other hand, this may also be attributed to the effective setting of cueing initial point in the experiment. In the cueing design, the purpose of setting TTC $=1.2 \mathrm{~s}$ was to match the passengers' motion anticipation and motion stimuli as much as possible in the time domain. This helps to enhance the 'sense of agency', thereby reducing passenger motion sickness. Therefore, in practical applications, it is important to guarantee the temporal matching between the cueing initial point and the real vehicle movement, which we think might be more important than magnitude matching.

As for questions 4 and 5 in Table 2, in the control condition, i.e., the participants without vibration cue, their answers about the upcoming motion and speed are a kind of 'hindsight'. To detail, their 'predictability and perceptibility' of the motion are only realized after the actual motion starts for a while or even has completely finished. By contrast, in the experiment condition the participants can generate motion anticipation with the help of vibration cues, which is a kind of 'foresight' and happens before or synchronously with the actual motion of lane change. This temporal difference of motion perception between the control and experiment conditions, corresponds to the difference between 'hindsight' and 'foresight', which is exactly the anchor point for the mitigation methodology of motion sickness via cueing. Compared to the significant results in question 4 , the experimental conditions also outperform the control conditions in question 5, but with no significant difference. This might indicate that for the studied scenario the cueing strategy in current form, though sufficient for enhancing motion anticipation of participants, cannot help them precisely predict the magnitude of upcoming motion. For applications in more complex scenarios, further ergonomic optimization of the cueing pattern is necessary.

\subsection{Mental workload and user-friendliness}

From the results of significant reduction of motion sickness degree, we find that the vibration cueing is similarly effective in mitigating sickness, compared to that of audio and visual cueing reported in previous studies. However, between the experiment condition and the control condition, we do not find significant difference in LF/HF results of participant HRV, which is not consistent with reference (Lin et al., 2011). A possible reason is that LF/HF is positively corelated to both sickness degree and mental workload (Lin et al., 2008). When the vibration cue system is on, motion sickness is reduced but the cognitive processing of cueing signal may increase the passenger mental workload. When the vibration cue system is off, motion sickness is severe but the passenger mental workload is low.

Typical non-driving tasks include making phone calls, reading, watching movies, etc., which mainly involve the use of visual or auditory perception. Comparing with visual or audio cueing solutions (Karjanto et al., 2018; Kuiper 
et al., 2020), the proposed vibration cue system provides information via the tactile perception, so the interference to non-driving tasks, if any, is expected to be little. However, the results of question 6 in Table 2 indicate for some passengers there are still negative effects on the ride. This may be due to the annoyances of vibration. Usually, all forms of cueing or warning systems, will more or less need a certain portion of cognition resources, which may increase their mental workload and accordingly affect non-driving task performances. In a research by Karjanto et al. (Karjanto et al., 2018), they suggested that although a given cue for the passengers engaging in NDT will increase their mental workload for the perception and cognition on the cue, if without any cue the passengers may still increase their mental workload in understanding the situation without enough certain information. Therefore, we think it is still unclear whether and how much the proposed vibration cue system brings any significant additional mental load to passengers engaging NDT. On the other hand, in the experiment setup a series of consecutive lane changes, and accordingly such frequent vibration cues appear more annoying. For normal applications in a real vehicle, there is no such frequent vibration, meaning that the unwanted disturbance or annoyance can be optimized.

\subsection{Needs for personalized cueing}

In question 3 of Table 2, some participants reported that the cueing should be given earlier, to allow more precise understanding of the cue and better preparation for the upcoming motion stimuli. As for question 7, not all participants thought the proposed vibration cue system was necessary for automated driving, showing that the prototype might not be a solution that fitted all participants. On one hand, we speculate that this may be due to the fact that the invited participants are not susceptible enough to motion sickness. To be specific, 6 out of 20 participants scored 2 for the necessity of the cueing system, while a 5 score means the most necessary. The average MSSQ score of these 6 participants is only $9.66 \pm 5.00$, which is lower than the overall average $12.06 \pm 6.14$, meaning that they are not susceptible to motion sickness. This result may be very different if we had invited sickness-susceptible participants.

On the other hand, this indicates that to realize better effects of sickness mitigation or user experience, it is necessary to offer a cueing system that can be personalized or even adaptive in setting. Such further improvements may include personalized optimization on a comprehensive decision strategy about when and how to initiate the cues, e.g. vibration intensity and cueing pattern, especially for complex motion stimuli. Certainly, for better user experience, the end users may freely set the cueing system based on their own preferences. Considering that vehicle acceleration with a changing direction is more likely to cause motion sickness than a constant-direction acceleration (Li et al., 2020), the direction change of future motion stimulus should be particularly presented in the cueing strategy. During a certain ride, the cueing strategy may be even adapted to the dynamic change of passenger motion sickness level, since there have been some studies on how to predict or estimate motion sickness level in real time. For example, a detailed vehicle-human model for sickness prediction was proposed by Salter et al. (2020). Based on the postural instability theory, Dong et al. (2011) pointed out that online monitoring of passenger postural activities might be useful to predict motion sickness level. Note that since there have been extensive studies on driver/occupant status monitoring in automated vehicles, e.g. using cabin cameras, it is promising to predict sickness via postural activities, which needs further practical application studies. Therefore, in order to achieve cueing strategy personalization for different passengers, besides their own susceptibility to motion sickness, the context information of the ride to be considered may further include their current mental or physical states, non-driving tasks, vehicle motion, traffic environment, etc. 
In principle, vibration cues may be provided with an equivalent or even larger bandwidth of cueing information than that of visual or audio cues, which is especially useful in fulfilling the cueing personalization. For example, the vibration motor array prototype can respond to a cueing command at more than $10 \mathrm{~Hz}$. The 4 by 4 array of vibration motors actually also supports more complex cueing forms for coupled multiple motions. These features allow much freedom of designing more comprehensive forms of cueing, such as the timing and patterns. Therefore, the proposed vibration cueing approach is promising for practical applications, while for best performances its detailed design should consider various acceptance and learning cost of different users.

\section{Conclusion}

To live up to our expectations of futuristic experience with self-driving, motion sickness should be well-mitigated in automated vehicles. A vibration cue system was proposed in this study, which could provide tactile notifications for the upcoming vehicle movements. A cushion-based prototype was fabricated and evaluated via driving simulator experiments. Results show that motion sickness could be significantly mitigated with the proposed cue system.

For further research, experiments with coupled lateral and longitudinal movement of vehicle can be designed, which can verify the effectiveness of vibration cue system in real applications. The experimental settings can be extended to explore the impact of vibration cues on the performance of participants' non-driving tasks, which may provide further evidences of the system user-friendliness. Additionally, for practical applications, the online personalization of cueing strategy should be studied, e.g. by real-time prediction of motion sickness level based on passenger postural activities.

\section{Acknowledgements}

This work was supported by Department of Science and Technology of Zhejiang (No. 2021C01SA601840, 2018C01058), and Ningbo Science \& Technology Bureau (No. 2018B10063, 2018B10064).

The authors appreciated our group members Biao Xu and Hao Pan for their help in carrying out the experiments, and Jiankan $\mathrm{Hu}$ for his help in providing the theoretical and experimental instructions.

\section{Supplementary materials}

A supplementary video abstract is provided, which includes a brief introduction of the proposed approach and validation experiments.

\section{ORCID iDs}

Daofei Li, https://orcid.org/0000-0002-6909-0169

Linhui Chen, https://orcid.org/0000-0002-8785-4783

\section{Declaration of interest statement}

No potential competing interest was reported by the authors. 


\section{References}

Bles, W., Bos, J.E., de Graaf, B., Groen, E., Wertheim, A.H., 1998. Motion sickness: only one provocative conflict? Brain Res. Bull. 47, 481-487. https://doi.org/10.1016/S0361-9230(98)00115-4

Bos, J.E., Bles, W., 1998. Modelling motion sickness and subjective vertical mismatch detailed for vertical motions. Brain Res. Bull. 47, 537-542. https://doi.org/10.1016/S0361-9230(98)00088-4

Bos, J.E., MacKinnon, S.N., Patterson, A., 2005. Motion sickness symptoms in a ship motion simulator: Effects of inside, outside, and no view. Aviat. Space Environ. Med. 76, 1111-1118.

Chang, C.-H., Stoffregen, T.A., Tseng, L.-Y., Lei, M.K., Cheng, K.B., 2021. Control of a virtual vehicle influences postural activity and motion sickness in pre-adolescent children. Hum. Mov. Sci. 78, 102832. https://doi.org/10.1016/j.humov.2021.102832

David, N., Newen, A., Vogeley, K., 2008. The "sense of agency" and its underlying cognitive and neural mechanisms. Conscious. Cogn. 17, 523-534.

Diels, C., Bos, J.E., 2016. Self-driving carsickness. Appl. Ergon. 53, 374-382. https://doi.org/10.1016/j.apergo.2015.09.009

Dong, X., Yoshida, K., Stoffregen, T.A., 2011. Control of a Virtual Vehicle Influences Postural Activity and Motion Sickness. J. Exp. Psychol.-Appl. 17, 128-138. https://doi.org/10.1037/a0024097

Golding, J.F., 2006. Predicting individual differences in motion sickness susceptibility by questionnaire. Personal. Individ. Differ. 41, 237-248. https://doi.org/10.1016/j.paid.2006.01.012

Karjanto, J., Md. Yusof, N., Wang, C., Terken, J., Delbressine, F., Rauterberg, M., 2018. The effect of peripheral visual feedforward system in enhancing situation awareness and mitigating motion sickness in fully automated driving. Transp. Res. Part F Traffic Psychol. Behav. 58, 678-692. https://doi.org/10.1016/j.trf.2018.06.046

Kuiper, Ouren X., Bos, J.E., Diels, C., Schmidt, E.A., 2020. Knowing what's coming: Anticipatory audio cues can mitigate motion sickness. Appl. Ergon. 85, 103068. https://doi.org/10.1016/j.apergo.2020.103068

Kuiper, Ouren X, Bos, J.E., Schmidt, E.A., Diels, C., Wolter, S., 2020. knowing what's coming: unpredictable motion causes more motion sickness. Hum. Factors 62, 1339-1348.

Li, D., Hu, J., 2021. Mitigating Motion Sickness in Automated Vehicles with Frequency-shaping Approach to Motion Planning. IEEE Robot. Autom. Lett. 6. https://doi.org/10.1109/LRA.2021.3101050

Li, D., Xu, B., Chen, L., Hu, Jiankan, 2021. Automated Car-following Algorithm Considering Passenger Motion Sickness. Presented at the The 6th International Symposium on Future Active Safety Technology Toward zero traffic accidents (FAST-zero'21), Kanazawa.

Li, Z., Fu, R., Wang, C., Stoffregen, T.A., 2020. Effects of Linear Acceleration on Passenger Comfort During Physical Driving on an Urban Road. Int. J. Civ. Eng. 18, 1-8. https://doi.org/10.1007/s40999-019-00473-8

Lin, C.-T., Lin, C.-L., Chiu, T.-W., Duann, J.-R., Jung, T.-P., 2011. Effect of respiratory modulation on relationship between heart rate variability and motion sickness, in: 2011 Annual International Conference of the IEEE Engineering in Medicine and Biology Society. Presented at the 2011 Annual International Conference of the IEEE Engineering in Medicine and Biology Society, pp. 1921-1924. https://doi.org/10.1109/IEMBS.2011.6090543

Lin, T., Imamiya, A., Mao, X., 2008. Using multiple data sources to get closer insights into user cost and task performance. Interact. Comput. 20, 364-374.

Malik, M., Bigger, J.T., Camm, A.J., Kleiger, R.E., Malliani, A., Moss, A.J., Schwartz, P.J., 1996. Heart rate variability: Standards of measurement, physiological interpretation, and clinical use. Eur. Heart J. 17, 354-381. https://doi.org/10.1093/oxfordjournals.eurheartj.a014868

Malińska, M., Zużewicz, K., Bugajska, J., Grabowski, A., 2015. Heart rate variability (HRV) during virtual reality immersion. Int. J. Occup. Saf. Ergon. 21, 47-54. https://doi.org/10.1080/10803548.2015.1017964

Meng, F., Spence, C., 2015. Tactile warning signals for in-vehicle systems. Accid. Anal. Prev. 75, $333-346$. https://doi.org/10.1016/j.aap.2014.12.013

Miksch, Markus, Steiner, M., Miksch, Michael, Meschtscherjakov, A., 2016. Motion Sickness Prevention System (MSPS): Reading Between the Lines, in: Adjunct Proceedings of the 8th International Conference on Automotive User Interfaces and Interactive Vehicular Applications, AutomotiveUI '16 Adjunct. Association for Computing Machinery, New York, NY, USA, pp. 147-152. https://doi.org/10.1145/3004323.3004340

Oman, C.M., 1990. Motion sickness: a synthesis and evaluation of the sensory conflict theory. Can. J. Physiol. Pharmacol. 68, 294-303. https://doi.org/10.1139/y90-044

Pfleging, B., Rang, M., Broy, N., 2016. Investigating user needs for non-driving-related activities during automated driving, in: Proceedings of the 15th International Conference on Mobile and Ubiquitous Multimedia, MUM '16. Association for Computing Machinery, New York, NY, USA, pp. 91-99. https://doi.org/10.1145/3012709.3012735

Reason, J.T., 1978. Motion sickness adaptation: A neural mismatch model. J. R. Soc. Med. 71, 819-829. https://doi.org/10.1177/014107687807101109 
Reason, J.T., 1969. Motion sickness - some theoretical considerations. Int. J. Man-Mach. Stud. 1, 21-38. https://doi.org/10.1016/S0020-7373(69)80009-X

Riccio, G.E., Stoffregen, T.A., 1991. An Ecological Theory of Motion Sickness and Postural Instability. Ecol. Psychol. 3, 195-240. https://doi.org/10.1207/s15326969eco0303_2

Rolnick, A., Lubow, R., 1991. Why is the driver rarely motion sick? The role of controllability in motion sickness. Ergonomics 34, 867-879.

Salter, S., Diels, C., Herriotts, P., Kanarachos, S., Thake, D., 2020. Model to predict motion sickness within autonomous vehicles. Proc. Inst. Mech. Eng. Part J. Automob. Eng. 234, 1330-1345. https://doi.org/10.1177/0954407019879785

Sivak, M., Schoettle, B., 2015. Motion sickness in self-driving vehicles. University of Michigan, Ann Arbor, Transportation Research Institute.

Ukita, R., Okafuji, Y., Wada, T., 2020. Lane-Change Control of Automated Vehicles to Reduce Motion Sickness Based on a Computational Model. https://doi.org/10.1109/SMC42975.2020.9283021

van Veen, T., Karjanto, J., Terken, J., 2017. Situation Awareness in Automated Vehicles through Proximal Peripheral Light Signals, in: Proceedings of the 9th International Conference on Automotive User Interfaces and Interactive Vehicular Applications. Presented at the AutomotiveUI '17: ACM 9th International Conference on Automotive User Interfaces and Interactive Vehicular Applications, ACM, Oldenburg Germany, pp. 287-292. https://doi.org/10.1145/3122986.3122993

Wada, T., 2016. Motion sickness in automated vehicles, in: Advanced Vehicle Control AVEC'16. CRC Press, pp. 169174. 